\title{
Nonadiabatic extension of the Heisenberg model
}

\author{
Ekkehard Krüger* \\ Max-Planck-Institut für Metallforschung, D-70506 Stuttgart, Germany
}

(Dated: November 17, 2018)

\begin{abstract}
The localized states within the Heisenberg model of magnetism should be represented by best localized Wannier functions forming a unitary transformation of the Bloch functions of the narrowest partly filled energy bands in the metals. However, as a consequence of degeneracies between the energy bands near the Fermi level, in any metal these Wannier functions cannot be chosen symmetry-adapted to the complete paramagnetic group $M^{P}$. Therefore, it is proposed to use Wannier functions with the reduced symmetry of a magnetic subgroup $M$ of $M^{P}$ [case (a)] or spin dependent Wannier functions [case (b)]. The original Heisenberg model is reinterpreted in order to understand the pronounced symmetry of these Wannier functions. While the original model assumes that there is exactly one electron at each atom, the extended model postulates that in narrow bands there are as many as possible atoms occupied by exactly one electron. However, this state with the highest possible atomiclike character cannot be described within the adiabatic (or Born-Oppenheimer) approximation because it requires a more realistic description of the electronic motion. Within the (true) nonadiabatic system the electrons move on localized orbitals that are still symmetric on the average of time, but not at any moment. These nonadiabatic states have the same symmetry as the adiabatic states and determine the commutation properties of the nonadiabatic Hamiltonian $H^{n}$. The nonadiabatic Heisenberg model is a purely group-theoretical model which interprets the commutation properties of $H^{n}$ that are explicitly given in this paper for the two important cases (a) and (b). There is evidence that the occurrence of these two types of Wannier functions in the band structure of a metal is connected with the occurrence of magnetism and superconductivity, respectively.
\end{abstract}

PACS numbers: Heisenberg model, 75.10.-b, 74.20.-z

\section{INTRODUCTION}

In any application of the Heisenberg model of magnetism, ${ }^{1}$ the localized states of the electrons should be represented by Wannier functions

$$
w_{i}\left(\vec{r}-\vec{R}-\vec{\rho}_{i}\right)=\frac{1}{\sqrt{N}} \sum_{\vec{k}}^{B Z} \sum_{q=1}^{\mu} e^{-i \vec{k}\left(\vec{R}+\vec{\rho}_{i}\right)} g_{i q}(\vec{k}) \varphi_{\vec{k} q}(\vec{r})
$$

with the following properties:

(i) the $w_{i}\left(\vec{r}-\vec{R}-\vec{\rho}_{i}\right)$ are centered on the atomic positions $\vec{R}+\vec{\rho}_{i}$;

(ii) the $w_{i}\left(\vec{r}-\vec{R}-\vec{\rho}_{i}\right)$ are gained by a unitary transformation from the Bloch functions $\varphi_{\vec{k} q}(\vec{r})$ of the energy bands of interest;

(iii) the $w_{i}\left(\vec{r}-\vec{R}-\vec{\rho}_{i}\right)$ are symmetry-adapted to the space group $G$ of the considered metal; and

(iv) the $w_{i}\left(\vec{r}-\vec{R}-\vec{\rho}_{i}\right)$ are as well localized as possible.

The first sum in Eq. (1.1) runs over the $N$ vectors $\vec{k}$ of the first Brillouin zone (BZ), the second sum runs over the $\mu$ bands of interest (with the band indices $q=1$ to $\mu$ ), and $\vec{R}$ and $\vec{\rho}_{i}$ denote the vectors of the Bravais lattice and the centers of symmetry of the Wannier functions within the unit cell, respectively.

The transformation is unitary [point (ii)] if the coefficients $g_{i q}(\vec{k})$ in Eq. (1.1) are the elements of a unitary matrix $\boldsymbol{g}(\vec{k})$,

$$
\boldsymbol{g}^{-1}(\vec{k})=\boldsymbol{g}^{\dagger}(\vec{k})
$$

The Wannier functions are symmetry-adapted to $G$ [point (iii)] if they satisfy the equation

$$
w_{i}\left(\alpha^{-1}\left(\vec{r}-\vec{R}-\vec{\rho}_{i}\right)\right)=\sum_{j=1}^{\mu} D_{j i}(\alpha) w_{j}\left(\vec{r}-\vec{R}-\vec{\rho}_{i}\right)
$$

for the elements $\alpha$ of the point group $G_{0}$ of $G$, where the matrices $\left[D_{j i}(\alpha)\right]$ form a (reducible or irreducible) singlevalued representation $D_{0}$ of $G_{0}$, see Eq. (1.8) of Ref. 2 . [Note that in Eq. (1.3) on the right hand side there is $\rho_{i}$ and not $\rho_{j}$.]

The Wannier functions are as well localizable as possible [point (iv)] if the Bloch-like functions

$$
\widetilde{\varphi}_{\vec{k} i}(\vec{r})=\sum_{q=1}^{\mu} g_{i q}(\vec{k}) \varphi_{\vec{k} q}(\vec{r})
$$

vary (for fixed $\vec{r}$ ) smoothly through the whole $\vec{k}$ space. ${ }^{2}$

It is one of the most important results of the group theory of Wannier functions that Wannier functions complying with all the four conditions given above exist only in isolated sets of $\mu$ energy bands which satisfy the grouptheoretical compatibility relations throughout the Brillouin zone. ${ }^{2,3,4,5,6}$ [This condition is necessary, but not sufficient: in addition, there must exist unitary matrices $\boldsymbol{S}(\vec{K})$ defined in Eq. (4.16) of Ref. 2 which satisfy the equations (4.17) and (4.28) of Ref. 2. These matrices $\boldsymbol{S}(\vec{K})$ determine the positions $\vec{\rho}_{i}$ of the Wannier functions.] In an "isolated" set of energy bands, each band may be connected by degeneracies to the other bands of 
this set, but must not be connected to bands not belonging to this set.

The mentioned "energy bands of interest" are the partly filled energy bands in the band structures of the considered metal. Often, it is only one roughly half-filled band which interests. However, the energy bands in the (paramagnetic) band structures of the metals are degenerate at several points and lines of symmetry of the Brillouin zone. Because of these degeneracies, is not possible to separate narrow isolated sets of energy bands which satisfy the compatibility relations throughout the Brillouin zone. For this reason, Wannier functions with all the properties demanded above do not exist in the metals.

Therefore, the localized states are often represented by "approximated" Wannier functions which no longer form an exactly unitary transformation of the Bloch functions. These Wannier functions are constructed from slightly modified energy bands in which some of the Bloch functions at points, lines, and planes of symmetry are replaced by Bloch functions with a symmetry appropriate for the construction of Wannier functions. Hence, these approximated Wannier functions have lost all the information connected with the symmetry of the removed Bloch functions and carry the wrong information of the new Bloch functions.

The nonadiabatic Heisenberg model (NHM) as proposed in this and previous papers ${ }^{7,8,9,10}$ extends the original Heisenberg model on the basis of Wannier functions which form an exactly unitary transformation of the Bloch functions of the bands of interest. Within this model, it is not allowed to replace any Bloch function in the calculated band structure by functions with a new symmetry. Hence, the NHM takes into account the complete information connected with the symmetry of the Bloch functions in the band structure of the considered metal.

Clearly, the Wannier functions used within the NHM cannot not comply with all the properties (i) - (iv) given above. The development of the nonadiabatic model was suggested by two observations:

1. An exactly unitary transformation of the Bloch functions of the partly filled bands into best localized Wannier functions becomes possible in nearly all the metals when the Wannier functions are allowed to have the reduced symmetry of a magnetic subgroup $M$ of the paramagnetic group [see appendix A, case (a)] or when they are allowed to be spin dependent [see appendix A, case (b)].

2. There is a deep connection between the symmetry of these exact Wannier functions in a metal and the physical properties of the electrons at the Fermi level..$^{7,8,9,10,11}$

The original Heisenberg model of magnetism is defined by the assumption that there is exactly one electron on each atom of a metal. The NHM replaces this assumption by introducing three postulates which will be given in the following Sec. II. These postulates combine in a new way the Heisenberg model with the band model. The fundamental second postulate given in Eq. (2.18) states that the probability to find exactly one electron on an atom is as large as possible in narrow energy bands.

The second postulate of the NHM cannot be satisfied within the adiabatic (or Born-Oppenheimer) approximation. In the framework of this approximation the electrons move in rigid orbitals in the average potential of the other electrons. The second postulate, however, requires a more realistic description of the electronic motion. In the true (nonadiabatic) system a localized electron moves in a potential depending on which of the adjacent localized states is occupied and on the present motion of the electrons in these states. These modified orbitals

$$
|\vec{T}, m, \nu\rangle
$$

are described by introducing a new quantum number $\nu$ which labels different states of motion of the center of mass of the localized states. ${ }^{12}[\vec{T}$ denotes the positions of the atoms, see Eq. (2.2).]

Nonadiabatic localized functions

$$
\langle\vec{r}, t, \vec{q} \mid \vec{T}, m, \nu\rangle
$$

(as introduced in the next section) which represent the nonadiabatic localized states are highly complicated. Hence, it will be practically impossible to give these functions explicitly. Fortunately, one important feature of these functions is known exactly: they have the same symmetry as the exact Wannier functions of the narrowest, roughly half-filled energy bands of the metal under consideration. Thus, any application of the NHM starts with a group-theoretical examination of the symmetry of the best localized (spin dependent) Wannier functions which is clearly determined by the symmetry of the Bloch functions in the band structure of the given metal. ${ }^{2,3,4,5,6,13}$ The symmetry of these Wannier functions is explicitly given for two important cases (a) and (b) in appendix A.

The NHM is a purely group-theoretical model. An explicit knowledge of the nonadiabatic localized functions (going beyond of their symmetry) does not provide new physical insight. Even in the nonadiabatic model, any calculation of expectation values should be carried out within the adiabatic approximation.

\section{NONADIABATIC HEISENBERG MODEL}

\section{A. General}

Consider a set of $\mu$ energy bands in a metal with the paramagnetic space group $G$, the paramagnetic group

$$
M^{P}=G+K G
$$

(with $K$ denoting the operator of time inversion), and $\mu$ atoms at the positions

$$
\vec{T}=\vec{R}+\vec{\varrho}_{i}
$$


per unit cell, where $\vec{R}$ and $\vec{\varrho}_{i}(i=1$ to $\mu)$ denote the vectors of the Bravais lattice and the positions of the $i$ th atom within the unit cell, respectively. The energy bands of this set are assumed to belong to the narrowest partly filled bands of this metal (while it is not demanded that all the narrow, partly filled bands belong to it).

Assume that the symmetry of the Bloch functions of the considered set of energy bands allows the construction of either Wannier functions

$$
w_{\vec{T} m}(\vec{r}, t) \equiv w_{i}\left(\vec{r}-\vec{R}-\vec{\rho}_{i}\right) u_{m}(t)
$$

symmetry-adapted to a magnetic subgroup $M$ of $M^{P}$ or spin dependent Wannier functions

$$
w_{\vec{T} m}(\vec{r}, t) \equiv w_{i m}\left(\vec{r}-\vec{R}-\vec{\rho}_{i}, t\right)
$$

symmetry-adapted to $M^{P}$. The former functions are defined appendix A, case (a), their symmetry is given in Eqs. (A17) and (A19), the latter are defined in appendix A, case (b), and their symmetry is given in Eqs. (A25) and (A26). The functions $u_{m}(t)$ are Pauli's spin functions, see Eq. (4.7), $t$ is the spin coordinate, and the (crystal) spin label $m= \pm \frac{1}{2}$ distinguished between the two functions at the same position $\vec{T}$. In either case, the Wannier functions form a unitary transformation of the exact Bloch functions of the considered set of $\mu$ energy bands, are situated on the atoms (with the positions $\vec{T})$, and are as well localized as possible.

\section{B. The three postulates of the nonadiabatic Heisenberg model}

Let be

$$
H=H_{H F}+H_{C b}
$$

the electronic Hamiltonian in the considered set of energy bands with $H_{H F}$ and

$$
\begin{aligned}
H_{C b}= & \sum_{\vec{T}, m}\left\langle\vec{T}_{1}, m_{1} ; \vec{T}_{2}, m_{2}\left|H_{C b}\right| \vec{T}_{1}^{\prime}, m_{1}^{\prime} ; \vec{T}_{2}^{\prime}, m_{2}^{\prime}\right\rangle \\
& \times c_{\vec{T}_{1} m_{1}}^{\dagger} c_{\vec{T}_{2} m_{2}}^{\dagger} c_{\vec{T}_{2}^{\prime} m_{2}^{\prime}} c_{\vec{T}_{1}^{\prime} m_{1}^{\prime}}
\end{aligned}
$$

representing the Hartree-Fock and Coulomb energy, respectively. The fermion operators $c_{\vec{T} m}^{\dagger}$ and $c_{\vec{T} m}$ create and annihilate electrons with (crystal) spin $m$ in the localized states $|\vec{T}, m\rangle$ represented by the Wannier functions $w_{\vec{T} m}(\vec{r}, t)$. Other contributions to $H$ from the electrons not belonging to the considered set of bands, are neglected even as are spin-orbit effects.

$H_{C b}$ may be written as

$$
H_{C b}=H_{c}+H_{e x}+H_{z},
$$

with the operator of Coulomb repulsion $H_{c}$ containing all the matrix elements of $H_{C b}$ with

$$
\vec{T}_{1}=\vec{T}_{1}^{\prime} \text { and } \vec{T}_{2}=\vec{T}_{2}^{\prime}
$$

the exchange operator $H_{e x}$ containing the matrix elements with

$$
\vec{T}_{1}=\vec{T}_{2}^{\prime} \text { and } \vec{T}_{2}=\vec{T}_{1}^{\prime},
$$

and $H_{z}$ comprising the remaining matrix elements, i.e., the matrix elements with

$$
\left\{\vec{T}_{1}, \vec{T}_{2}\right\} \neq\left\{\vec{T}_{1}^{\prime}, \vec{T}_{2}^{\prime}\right\} .
$$

The interaction $H_{z}$ is of great importance within the NHM. In order to discuss the effect of $H_{z}$, consider the operator

$$
H^{\prime}=H_{H F}+H_{c}+H_{e x}
$$

obtained from the complete Hamiltonian $H$ by putting $H_{z}=0$.

As is well-known, the Coulomb repulsion of two electrons occupying localized states at the same atom is larger than the Coulomb repulsion of two electrons at different atoms. ${ }^{14}$ The electronic motion in the ground state $\left|G^{\prime}\right\rangle$ of $H^{\prime}$ has an "atomiclike" character when the Coulomb repulsion between the localized states determines the electronic motion in $\left|G^{\prime}\right\rangle$ to such an extend that the probability to find two electrons (with different spin directions) on the same atom is markedly smaller than in case of a purely bandlike motion. In this context, I speak of a "purely bandlike" motion when the probability to find an electron in the localized state $|\vec{T} m\rangle$ is independent of whether or not the other state $|\vec{T},-m\rangle$ is occupied. In this case, the ground state consists of configurations with nearly random occupation.

The atomic- or bandlike character depends on the mean time of stay $\tau \approx \hbar / \Delta$ of the electrons at the atoms and, hence, on the bandwidth $\Delta$. For $\Delta \rightarrow 0$ we have $\tau \rightarrow \infty$; the metal becomes a Mott insulator representing a perfectly atomiclike state. For $\Delta \rightarrow \infty$, on the other hand, we have $\tau \rightarrow 0$ and, hence, a purely bandlike character of the electrons. Thus, as is well-known, the electrons in partly filled energy bands tend to a more atomiclike behavior with decreasing bandwidth and to a more bandlike behavior with increasing bandwidth. ${ }^{15}$

Now assume the considered energy bands to be sufficiently narrow that the ground state $\left|G^{\prime}\right\rangle$ of $H^{\prime}$ clearly has atomiclike character. The matrix elements of $H_{z}$ satisfy neither Eq. (2.8) nor (2.9). Thus, the interaction $H_{z}$ annihilates two electrons in localized states at the positions $\vec{T}_{1}^{\prime}$ and $\vec{T}_{2}^{\prime}$ and creates at least one of them at the new positions $\vec{T}_{1}$ or $\vec{T}_{2}$. Hence, unlike $H_{c}$ or $H_{e x}$, the operator $H_{z}$ generates transitions between adjacent localized states which lead to configurations with a more random occupation. Consequently, the probability to find two electrons at the same position $\vec{T}$ will be larger in the ground state $|G\rangle$ of the complete Hamiltonian

$$
H=H^{\prime}+H_{z}
$$

[given in Eq. (2.5)] than in the ground state $\left|G^{\prime}\right\rangle$ of $H^{\prime}$. Therefore, the total Coulomb repulsion energy in $|G\rangle$ is 
larger than in $\left|G^{\prime}\right\rangle$ and we may assume that in sufficiently narrow bands the ground state energy $E$ of $H$ is greater than the ground state energy $E^{\prime}$ of $H^{\prime}$.

Still "sufficiently" narrow means that the Coulomb repulsion between the localized states determines the electronic motion in $\left|G^{\prime}\right\rangle$. It is difficult to decide how narrow such sufficiently narrow bands should be. However, we know that there is strong theoretical and experimental evidence that, e.g., the $d$ electrons of the transition metals exhibit behavior of both the band and the Heisenberg model. ${ }^{15}$ Therefore, we may suppose that $E>E^{\prime}$ is valid in the narrowest bands of the metals. This supposition leads to the first postulate of the NHM.

Postulate 1 In the narrowest, partly filled energy bands of the metals the transitions generated by $H_{z}$ are energetically unfavorable, i.e., we have

$$
\langle G|H| G\rangle>\left\langle G^{\prime}\left|H^{\prime}\right| G^{\prime}\right\rangle,
$$

where $|G\rangle$ and $\left|G^{\prime}\right\rangle$ denote the exact ground states of $H$ and $H^{\prime}$, respectively.

The particular form of the matrix elements of $H_{z}$ shows that it represents a short-ranged interaction which crucially depends on the exact form of the localized functions. This fact suggests that only small changes of the localized electronic orbitals are required to prevent the transitions generated by $H_{z}$. However, such modified orbitals do not exist within the adiabatic approximation because such modifications yield localized charge distributions which are not symmetric at any moment. As a consequence, the nuclei become accelerated in varying directions. Hence, we replace the (adiabatic) localized states (represented by the Wannier functions) by more realistic nonadiabatic localized states

$$
|\vec{T}, m, \nu\rangle
$$

which take into account the motion of the nuclei. The new quantum number $\nu$ labels different states of motion of the center of mass of the nucleus and the electron occupying the state $|\vec{T}, m, \nu\rangle .^{12}$

The nonadiabatic Hamiltonian $H^{n}$ may be written as

$$
H^{n}=H_{H F}+H_{C b}^{n}
$$

where the Coulomb interaction now has the form

$$
\begin{aligned}
H_{C b}^{n}= & \sum_{\vec{T}, m}\left\langle\vec{T}_{1}, m_{1}, n ; \vec{T}_{2}, m_{2}, n\left|H_{C b}\right| \vec{T}_{1}^{\prime}, m_{1}^{\prime}, n ; \vec{T}_{2}^{\prime}, m_{2}^{\prime}, n\right\rangle \\
& \times c_{\vec{T}_{1} m_{1}}^{n \dagger} c_{\vec{T}_{2} m_{2}}^{n \dagger} c_{\vec{T}_{2}^{\prime} m_{2}^{\prime}}^{n} c_{\vec{T}_{1}^{\prime} m_{1}^{\prime}}^{n} .
\end{aligned}
$$

The new fermion operators $c_{\vec{T} m}^{n \dagger}$ and $c_{\vec{T} m}^{n}$ create and annihilate electrons with crystal spin $m$ [see appendix A, case (b)] in the nonadiabatic localized states $|\vec{T}, m, n\rangle$.
The matrix elements of $H_{C b}^{n}$ are integrals

$$
\begin{aligned}
& \left\langle\vec{T}_{1}, m_{1}, n ; \vec{T}_{2}, m_{2}, n\left|H_{C b}\right| \vec{T}_{1}^{\prime}, m_{1}^{\prime}, n ; \vec{T}_{2}^{\prime}, m_{2}^{\prime}, n\right\rangle \\
& =\frac{e^{2}}{2} \sum_{t t^{\prime}} \int\left\langle\vec{T}_{1}, m_{1}, n \mid \vec{r}, t, \vec{q}\right\rangle\left\langle\vec{T}_{2}, m_{2}, n \mid \vec{r}^{\prime}, t^{\prime}, \vec{q}^{\prime}\right\rangle \\
& \quad \times \frac{1}{\left|\vec{r}-\vec{r}^{\prime}\right|}\left\langle\vec{r}, t, \vec{q} \mid \vec{T}_{1}^{\prime}, m_{1}^{\prime}, n\right\rangle\left\langle\vec{r}^{\prime}, t^{\prime}, \vec{q}^{\prime} \mid \vec{T}_{2}^{\prime}, m_{2}^{\prime}, n\right\rangle \\
& \quad \times d \vec{r} d \vec{r}^{\prime} d \vec{q} d \vec{q}^{\prime}
\end{aligned}
$$

over nonadiabatic localized functions of the form

$$
\langle\vec{r}, t, \vec{q} \mid \vec{T}, m, n\rangle
$$

where $\nu=n$ labels the nonadiabatic states which satisfy the following Eq. (2.18), and the new coordinate $\vec{q}$ stands for that part of the motion of the center of mass of the localized state $|\vec{T}, m, n\rangle$ which nonadiabatically follows the motion of the electron occupying this state. We may imagine that $\vec{q}$ denotes the acceleration of the nucleus (together with the core electrons).

Within the nonadiabatic localized states $|\vec{T}, m, \nu\rangle$ the electrons possess considerably more room to move than within the adiabatic states. While in the adiabatic approximation the symmetry operators $P(a)$ act on $\vec{r}$ and $t$ alone, in the nonadiabatic system these operators act on $\vec{r}, t$, and the acceleration $\vec{q}$ of the nuclei, see Eq. (B9). The nonadiabatic localized functions have no definite transformation properties under space group operations acting only on $\vec{r}$ and $t$. Hence, the symmetry of the adiabatic and nonadiabatic localized states [given by Eq. (B1) for the fermion operators] may be interpreted as follows.

Within the adiabatic system the electrons move on orbitals being symmetric with respect to the lattice at any moment. Within the nonadiabatic system, on the other hand, the orbitals are still symmetric on the average of time, but not at any moment. This statement is independent of the absolute value $|\vec{q}|$ of the acceleration of the nuclei, i.e., it is independent of whether or not the mass of the electrons is markedly smaller than the mass of the nuclei.

Thus, the introduction of the new quantum number $\nu$ allows the electrons to move in a potential depending on which of the adjacent localized states are occupied and on the present positions of these electrons. Hence, within the nonadiabatic system the electrons should be able to avoid the transitions generated by $H_{z}$ by an appropriately modified motion, if these transitions are energetically unfavorable, i.e., if the relation (2.12) is true. Thus, as a consequence of relation (2.12), all the matrix elements of $H_{C b}^{n}$ which neither satisfy Eq. (2.8) nor Eq. (2.9) should vanish.

For this reason, we suppose that the transitions generated by $H_{z}$ are artifacts of the adiabatic approximation and do not happen in the (true) nonadiabatic system if relation (2.12) is satisfied. This supposition leads to the second postulate of the NHM. 
Postulate 2 If relation (2.12) is true, the Coulomb interaction $H_{\mathrm{Cb}}^{n}$ does not generate transitions between adjacent localized states, i.e.,

$$
\left\langle\vec{T}_{1}, m_{1}, n ; \vec{T}_{2}, m_{2}, n\left|H_{C b}\right| \vec{T}_{1}^{\prime}, m_{1}^{\prime}, n ; \vec{T}_{2}^{\prime}, m_{2}^{\prime}, n\right\rangle=0
$$

for

$$
\left\{\vec{T}_{1}, \vec{T}_{2}\right\} \neq\left\{\vec{T}_{1}^{\prime}, \vec{T}_{2}^{\prime}\right\}
$$

and for special nonadiabatic localized functions

$$
\langle\vec{r}, t, \vec{q} \mid \vec{T}, m, n\rangle
$$

labeled by $\nu=n$.

At the transition from the adiabatic to the nonadiabatic system, the total energy of the electron system decreases by

$$
\Delta E=\langle G|H| G\rangle-\left\langle G^{\prime}\left|H^{\prime}\right| G^{\prime}\right\rangle
$$

if we neglect the energy of the nonadiabatic motion of the nuclei and the energy change caused by the slight modification of the electronic orbitals within the nonadiabatic states.

As a consequence of Eq. (2.18), the commutation properties of the operator $H_{C b}^{n}$ depend on the symmetry of the nonadiabatic localized states. Since only small modifications of the adiabatic electronic orbitals are required to prevent the transitions generated by $H_{z}$, we can assume that the nonadiabatic Hamiltonian $H^{n}$ has the same commutation properties as the adiabatic Hamiltonian $H^{\prime}$ given in Eq. (2.11). This is the third (and last) postulate of the NHM.

Postulate 3 If relation (2.12) is true, the nonadiabatic Hamiltonian $H^{n}$ has the same commutation properties as the adiabatic Hamiltonian $H^{\prime}$, i.e.,

$$
\left[H^{\prime}, P\right]\left\{\begin{array}{l}
= \\
\neq
\end{array}\right\} 0 \Rightarrow\left[H^{n}, P\right]\left\{\begin{array}{l}
= \\
\neq
\end{array}\right\} 0,
$$

where $P$ stands for any symmetry operator.

As a consequence, the nonadiabatic localized functions have the same symmetry as the (adiabatic) Wannier functions $w_{\vec{T} m}(\vec{r}, t)$.

\section{SYMMETRY OF THE OPERATOR $H^{\prime}$}

According to its definition, the operator $H^{\prime}$ arises from the complete adiabatic Hamiltonian $H$ in Eq. (2.5) by putting

$$
H_{z}=0 .
$$

This equation does not state that $H_{z}$ is neglected, but that $H_{z}$ is put equal to zero. By this step, the commutation properties of the operator $H^{\prime}$ depend on the symmetry of the Wannier functions, whereas the commutation properties of the complete adiabatic Hamiltonian $H$ are independent of the symmetry of the used basis functions. The nonadiabatic matrix elements of $H_{z}$, however, vanish within the NHM, see Eq. (2.18).

\section{Case (a): The Wannier functions are symmetry-adapted to a magnetic group}

If the Wannier functions are symmetry-adapted only to a magnetic subgroup $M$ of the paramagnetic group $M^{P}$, the symmetry of the operator $H^{\prime}$ is given by

$$
\left[H^{\prime}, P(a)\right]=0 \quad \text { for } a \in M
$$

and

$$
\left[H^{\prime}, P(a)\right] \neq 0 \quad \text { for } a \in\left(M^{P}-M\right),
$$

where

$$
M^{P}=G+K G
$$

stands for the paramagnetic group (and $G$ is the space group). The symmetry operators $P(a)$ are given in Eq. (A3) and $K$ denotes the operator of time inversion. Especially, in this case (a) $H^{\prime}$ does not commute with $K$,

$$
\left[H^{\prime}, K\right] \neq 0,
$$

since $K \in\left(M^{P}-M\right)$.

The first equation (3.2) is valid since the complete Hamiltonian $H$ commutes with $P(a)$ and also the operator $P(a) H^{\prime} P^{-1}(a)$ complies with Eqs. (2.8) and (2.9) if $a \in M$ since the fermion operators $P(a) c_{\vec{T} m}^{\dagger} P^{-1}(a)$ and $P(a) c_{\vec{T} m} P^{-1}(a)$ are for all the $a \in M$ linear combinations of operators $c_{\vec{T}^{\prime} m^{\prime}}^{\dagger}$ and $c_{\vec{T}^{\prime} m^{\prime}}$, respectively, labeled by the same position $\vec{T}^{\prime}$, see the equations (B1) and (B2).

Within the NHM it is important that $H^{\prime}$ does not commute with $P(a)$ for $a \in\left(M^{P}-M\right)$. In the case (a) considered in this section, the Wannier functions cannot be chosen in such a way that they are symmetry-adapted to a group $\widehat{M}$ containing the operation $a$ as well as all the elements of $M$. Consequently, for $a \in\left(M^{P}-M\right)$, the fermion operators $P(a) c_{\vec{T} m}^{\dagger} P^{-1}(a)$ do not comply with Eq. (B1) or (B2), but are linear combinations

$$
P(a) c_{\vec{T} m}^{\dagger} P^{-1}(a)=\sum_{\overrightarrow{T^{\prime} m^{\prime}}} d_{\vec{T}^{\prime} m^{\prime}, \vec{T} m}(\alpha) c_{\vec{T}^{\prime} m^{\prime}}^{\dagger}
$$

of at least two operators $c_{\vec{T}^{\prime} m^{\prime}}^{\dagger}$ with different labels $\vec{T}_{1}^{\prime}$ and $\vec{T}_{2}^{\prime}$. We show that therefore the operator $P(a) H^{\prime} P^{-1}(a)$ has matrix elements violating Eq. (2.8) or (2.9).

Consider a fermion operator combination belonging to the Coulomb interaction of $H^{\prime}$, say

$$
O=c_{\vec{T}_{1}}^{\dagger} c_{\vec{T}_{2}}^{\dagger} c_{\vec{T}_{2}} c_{\vec{T}_{1}}
$$


and assume for a special $a \in\left(M^{P}-M\right)$ the sum in Eq. (3.5) to consist of two summands,

$$
P c_{\vec{T}}^{\dagger} P^{-1}=a \cdot c_{\vec{U}}^{\dagger}+b \cdot c_{\vec{V}}^{\dagger},
$$

labeled by the different positions $\vec{U}$ and $\vec{V}$. In Eq. (3.7) we use the abbreviation $P \equiv P(a)$ and drop the index $m$ since it does not matter here. With Eq. (3.7) we obtain

$$
\begin{aligned}
P O P^{-1}= & P c_{\vec{T}_{1}}^{\dagger} P^{-1} P c_{\vec{T}_{2}}^{\dagger} P^{-1} P c_{\vec{T}_{2}} P^{-1} P c_{\vec{T}_{1}} P^{-1} \\
= & \left(a c_{\vec{U}_{1}}^{\dagger}+b c_{\vec{V}_{1}}^{\dagger}\right)\left(a c_{\vec{U}_{2}}^{\dagger}+b c_{\vec{V}_{2}}^{\dagger}\right) \\
& \times\left(a^{*} c_{\vec{U}_{2}}+b^{*} c_{\vec{V}_{2}}\right)\left(a^{*} c_{\vec{U}_{1}}+b^{*} c_{\vec{V}_{1}}\right)
\end{aligned}
$$

where $a \neq 0$ and $b \neq 0$. For instance, the operator product

$$
c_{\vec{U}_{1}}^{\dagger} c_{\vec{V}_{2}}^{\dagger} c_{\vec{U}_{2}} c_{\vec{U}_{1}}
$$

belonging to $P O P^{-1}$ and, hence, to $P H^{\prime} P^{-1}$, violates Eq. (2.8) since $\vec{V}_{2} \neq \vec{U}_{2}$. Consequently, $H^{\prime}$ does not commute with $P$ as expressed by Eq. (3.3). In the same way, $H^{\prime}$ does not commute with $P$ when there are more than two summands on the right hand side of Eq. (3.7).

Case (b): The Wannier functions are spin dependent and symmetry-adapted to the paramagnetic group

If we consider spin dependent Wannier functions symmetry-adapted to the paramagnetic group $M^{P}$, then we have

$$
\left[H^{\prime}, P(a)\right]=0 \quad \text { for } a \in M^{P}
$$

and, especially,

$$
\left[H^{\prime}, K\right]=0 .
$$

However, in this case (b), the operator $H^{\prime}$ has matrix elements with

$$
m_{1}+m_{2} \neq m_{1}^{\prime}+m_{2}^{\prime}
$$

because the coefficients $f_{s m}(q, \vec{k})$ in Eq. (A21) cannot be chosen independent of $\vec{k}$, see appendix A, case (b). Therefore, $H^{\prime}$ does not conserve the crystal spin and, hence ${ }^{16}$, does not commute with the operators $M(\alpha)$ of the crystal spin defined in Eq. (A30),

$$
\left[H^{\prime}, M(\alpha)\right] \neq 0
$$

for at least one $\alpha \in G_{M} \cdot{ }^{17}$

\section{SYMMETRY OF THE NONADIABATIC HAMILTONIAN $H^{n}$}

\section{A. Magnetic and paramagnetic group}

The nonadiabatic Hamiltonian $H^{n}$ has the same commutation properties as the adiabatic operator $H^{\prime}$, see
Eq. (2.20). However, the symmetry operators $P(a)$ now act on $\vec{r}, t$, and on the new coordinate $\vec{q}$ of the nonadiabatic localized functions, see Eq. (B9).

Hence, we have

$$
\begin{gathered}
{\left[H^{n}, P(a)\right]=0 \quad \text { for } a \in M,} \\
{\left[H^{n}, P(a)\right] \neq 0 \quad \text { for } a \in\left(M^{P}-M\right),}
\end{gathered}
$$

and, especially,

$$
\left[H^{n}, K\right] \neq 0
$$

in the case (a) of the preceding section III, and

$$
\left[H^{n}, P(a)\right]=0 \quad \text { for } a \in M^{P},
$$

especially,

$$
\left[H^{n}, K\right]=0
$$

in the case (b) of the preceding section III.

\section{B. Crystal spin}

The nonadiabatic fermion operators in Eq. (2.14) are no longer labeled by the spin quantum number $s$. Hence, within the nonadiabatic system, the exact Fermi excitations are no longer purely electronic states but localized states of well-defined symmetry which are occupied by electrons carrying with them some nonadiabatic motion of the nuclei.

Let be $S(\alpha)$ with

$S(\alpha) u_{s}(t) \equiv u_{s}\left(\alpha^{-1} t\right)=\sum_{s^{\prime}} d_{s^{\prime} s}(\alpha) u_{s^{\prime}}(t) \quad$ for $\alpha \in O(3)$

the operators turning the electron spin, where the functions

$$
u_{s}(t)=\delta_{s t}
$$

are Pauli's spin functions with the spin quantum number $s= \pm \frac{1}{2}$ and the spin coordinate $t= \pm \frac{1}{2}$, and the matrices $\left[d_{s^{\prime} s}(\alpha)\right]$ are the representatives of the two-dimensional double-valued representation $D_{1 / 2}$ of the three-dimensional rotation group $O(3)$.

The adiabatic Hamiltonian $H$ given in Eq. (2.5) commutes with the operators $S(\alpha)$,

$$
[H, S(\alpha)]=0 \quad \text { for } \alpha \in O(3) .
$$

This equation expresses the conservation law of the spin angular momentum within the adiabatic system.

The nonadiabatic Hamiltonian $H^{n}$, on the other hand, does not commute with the operators $S(\alpha)$ (for $\alpha \neq E$ ) since the nonadiabatic fermion operators are no longer labeled by the spin quantum number $s$. Hence, as a consequence of the (small) shift of the Fermi character at the 
transition from the adiabatic to the nonadiabatic system, the electron spin angular momentum is no longer a conserved quantity. Now there exists an interaction between the electron spins and the nonadiabatic motion of the nuclei.

However, even in the nonadiabatic system there should exist a conserved quantity related to the conservation law of angular momentum. Thus, the equation (4.8) should be replaced by an analogous equation

$$
\left[\mathcal{H}^{n}, M(\alpha)\right]=0 \quad \text { for } \alpha \in G_{M}
$$

in the nonadiabatic system, where $\mathcal{H}^{n}$ stands for the complete nonadiabatic Hamiltonian. The group $G_{M}$ and the operators $M(\alpha)$ are defined in Eqs. (A29) and (A30). They act on the quantum number $m$ of the nonadiabatic localized states $|\vec{T}, m, n\rangle$ in the same manner as the operators $S(\alpha)$ act on the spin quantum number $s$ of Pauli's spin functions $u_{s}(t)$, cf. Eq. (B3). Therefore, these operators may be called the symmetry operators of the "crystal spin" and $m$ may be called the quantum number of the crystal spin. This is in analogy to the wave vector $\vec{k}$ of the Bloch functions which is sometimes referred to as "crystal momentum" in order to distinguish it from the momentum $\vec{p}$.

In the case (b), i.e., if we consider spin dependent Wannier functions symmetry-adapted to the paramagnetic group $M^{P}$, the adiabatic operator $H^{\prime}$ does not commute with all the operators $M(\alpha)$, see Eq. (3.12). Hence, also the nonadiabatic Hamiltonian $H^{n}$ as defined in Eq. (2.14) does not conserve the crystal spin,

$$
\left[H^{n}, M(\alpha)\right] \neq 0
$$

for at least one $\alpha \in G_{M} \cdot{ }^{17}$ It is one of the most interesting problems of the NHM to interpret this equation, see Sec. VB, case (b).

\section{DISCUSSION}

\section{A. Crystal electrons}

The NHM has been developed in order to interpret the symmetry and spin dependence of the Wannier functions in metals. These Wannier functions form an exactly unitary transformation of the Bloch functions of a set of partly filled energy bands in the band structure of the metal of interest.

In the framework of the adiabatic approximation, Wannier functions form nothing but a unitary basis within the considered bands. Hence, the commutation properties of the adiabatic Hamiltonian $H$ are independent of the symmetry of the Wannier functions, since the symmetry of any adiabatic Hamiltonian is independent of the symmetry of the used basis functions. The symmetry and localization of the Wannier functions simplifies the calculation of the matrix elements of $H$, but has no further physical meaning.
The nonadiabatic Hamiltonian $H^{n}$, on the other hand, has an important feature which distinguishes it from any adiabatic Hamiltonian $H$ : the commutation properties and the spin dependence of $H^{n}$ depend on the symmetry and spin dependence of the nonadiabatic localized functions. This is because the nonadiabatic localized functions have a physical meaning going beyond the meaning of pure basis functions: they represent states that are really occupied by the electrons in the way described by Mott $^{14}$ and Hubbard ${ }^{15}$ : the electrons occupy the nonadiabatic localized states as long as possible and perform their band motion by hopping from one atom to another. Such a band motion is generally referred to as atomiclike motion.

Within the NHM we may extend this picture of the atomiclike electron. Here the whole localized state $|\vec{T}, m, n\rangle$ behaves like a moving particle, say "crystal electron", with the local coordinate $\vec{T}$ and the crystal spin $m$. The spatial extend of the crystal electron is determined by the charge distribution of the localized state and the crystal spin is a conserved quantity.

First consider the picture of the crystal electron within the adiabatic approximation. Both operators $H_{c}$ and $H_{e x}$ [given in Eq. (2.7)] represent interactions between crystal electrons and, hence, are in accordance with this picture. The interaction $H_{z}$, on the other hand, contradicts the picture of a moving crystal electron because it destroys these new particles. Since $H_{z}$ is a short-ranged interaction, we may say that within the adiabatic system "the crystal electrons become destroyed at the slightest touch".

Within the NHM, on the other hand, Eq. (2.18) is valid. The crystal electrons are stable in the nonadiabatic system because the Coulomb interaction does not generate transitions between adjacent localized states. We may interpret Eq. (2.18) by stating that "the crystal electrons become slightly deformed but not destroyed at a touch". The crystal electrons now have a certain elasticity protecting them from being destroyed at any collision. In this context, the stability of crystal electrons increases with decreasing band width.

\section{B. Outlook}

The symmetry of the nonadiabatic Hamiltonian $H^{n}$ is given in Sec. IV for two interesting cases (a) and (b) which shall be considered separately.

\section{Case (a): The Wannier functions are symmetry-adapted to a magnetic group}

Sets of narrow, roughly half-filled energy bands with Wannier functions symmetry-adapted to a magnetic group $M$ as defined in the appendix A, case (a), have already been identified in the paramagnetic band structures of iron ${ }^{9}$ and chromium ${ }^{8}$. In both metals, this set 
consists of one "magnetic" band. In iron the related magnetic group

$$
M=I 4 / m m^{\prime} m^{\prime}=C_{4 h}^{5}+K\left\{C_{2 x} \mid \overrightarrow{0}\right\} C_{4 h}^{5}
$$

is the group of the ferromagnetic state and in chromium

$$
M=P_{I} 4 / m n c=D_{4 h}^{6}+K\{E \mid \tau\} D_{4 h}^{6}
$$

is the group of the commensurate spin-density-wave state.

In the case (a) considered in this section, the symmetry of the nonadiabatic Hamiltonian $H^{n}$ is given by the Eqs. (4.1), (4.2), and (4.3). Equation (4.3) shows that $H^{n}$ does not commute with the operator $K$ of time inversion. Therefore, $H^{n}$ cannot have a paramagnetic or superconducting ground state, since both states are invariant with respect to the time inversion.

Thus, the electrons of the magnetic band may gain the energy $\Delta E$ [given in Eq. (2.19)] only if the electron spins form a structure with the magnetic group $M$. This fact may be interpreted as follows. 8,9

The electrons of the magnetic band activate a spin dependent exchange mechanism producing a spin structure with the space group $M$. This is possible since, first, the electrons can modify their orbitals in the nonadiabatic localized states and, secondly, exchange integrals depend very sensitively on the exact form of the electronic orbitals. Hence, the electrons of the magnetic band modify their orbitals in such a way that the exchange energy $E_{e x}$ is maximum for a spin structure with the group $M$.

The condensation energy $E_{f}$, i.e., the energy difference between the paramagnetic and the magnetic state, is no longer given by the exchange energy $E_{e x}$ alone, but by

$$
E_{f}=\Delta E+E_{e x} .
$$

Hence, $E_{f}$ may be positive even if $E_{e x}$ is negative.

Case (b): The Wannier functions are spin dependent and symmetry-adapted to the paramagnetic group

Sets of narrow, roughly half-filled energy bands with spin dependent Wannier functions symmetry-adapted to the paramagnetic group $M^{P}$ as defined in the appendix A, case (b), have already been identified in the band structures of a great number of superconductors. ${ }^{7,10,11}$ It is remarkable that such "superconducting" bands cannot be found in those metals (such as $\mathrm{Li}, \mathrm{Na}, \mathrm{K}$, $\mathrm{Rb}, \mathrm{Cs}, \mathrm{Ca} \mathrm{Cu}, \mathrm{Ag}$, and $\mathrm{Au}$ ) which do not become superconducting. ${ }^{11}$

In the case (b) considered in this section, the symmetry of the nonadiabatic Hamiltonian $H^{n}$ is given by the Eqs. (4.4), (4.5), and (4.10). Eqs. (4.4) and (4.5) show that the ground state of $H^{n}$ has the correct symmetry of the paramagnetic group $M^{P}$. Especially, $H^{n}$ commutes with the operator $K$ of time inversion. Therefore, $H^{n}$ may have a paramagnetic or a superconducting ground state, but has not a magnetic ground state.
Equation (4.10) shows that $H^{n}$ does not conserve the crystal spin angular momentum. Hence, the electrons of the considered bands may gain the energy $\Delta E$ [given in Eq. (2.19)] only if they couple to other excitations in such a way that the conservation of the crystal spin angular momentum is satisfied in the nonadiabatic system. This fact may be interpreted as follows. ${ }^{7,18,19}$

In isotropic materials, the electrons of the considered bands couple to the phonons. This is possible since, first, the symmetry of localized acoustic phonons shows that they are able to carry crystal spin angular momentum, secondly, the electron spins are coupled to the phonons via the nonadiabatic motion of the nuclei, and, thirdly, the resulting nonadiabatic Hamiltonian complies with the conservation law of crystal spin angular momentum.

In anisotropic materials (consisting of one- or twodimensional sublattices), phonons are not able to transport crystal spin angular momenta through the crystal. Here the electrons of the considered bands are forced to couple to energetically higher-lying boson excitations.

At zero temperature, this spin-boson interaction constrains the electrons of the considered bands in a new way to form Cooper pairs because the conservation of spin angular momentum would be violated in any normal conducting state. Apart from this participation of the conservation of spin angular momentum, the mechanism of Cooper pair formation within the NHM is identical to the familiar mechanism presented within the BardeenCooper-Schrieffer (BCS) theory. ${ }^{20}$

The participation of the conservation law of spin angular momentum may be interpreted in terms of quantum mechanical constraining forces which constrain the electrons to form Cooper pairs. There is evidence that these constraining forces are necessary for the Hamiltonian to have eigenstates in which the electrons form Cooper pairs.

If this is true, then the BCS theory of superconductivity is only applicable to superconducting bands as defined in the appendix A, case (b). When it is applied to other bands, it does not yield the absolute energy minimum in the Hilbert space.

\section{Acknowledgments}

I am indebted to Ernst Helmut Brandt for stimulating discussions on the new model.

\section{APPENDIX A: SYMMETRY-ADAPTED WANNIER FUNCTIONS}

Consider a metal with the space group $G$ and the point group $G_{0}$. The elements

$$
a=\{\alpha \mid \vec{t}\}
$$


of $G$ consist of a point group operation $\alpha$ and a translation vector

$$
\vec{t}=\vec{\tau}(\alpha)+\vec{R}
$$

which is the sum of the nonprimitive translation $\vec{\tau}(\alpha)$ associated with $\alpha$ and a translation vector $\vec{R}$ of the Bravais lattice.

The operators $P(a)$ act on a wave function $f(\vec{r}, t)$ depending on the position $\vec{r}$ and the spin coordinate $t$ according to

$$
P(a) f(\vec{r}, t)=f\left(\alpha^{-1} \vec{r}-\alpha^{-1} \vec{t}, \alpha^{-1} t\right),
$$

where the symbol $\alpha^{-1} t$ is defined in Eq. (4.6).

The effect of $K$ is given by the equations ${ }^{21}$

$$
K f(\vec{r})=f^{*}(\vec{r}),
$$

where $f(\vec{r})$ stands for any function of position, and

$$
K u_{s}(t)=g_{s} u_{-s}(t),
$$

with $^{22}$

$$
g_{ \pm 1 / 2}=\mp i
$$

\section{Case (a): Wannier functions symmetry-adapted to a magnetic group}

Consider a set of $\mu$ energy bands in the paramagnetic band structure of a metal with $\mu$ atoms per unit cell. The positions of the atoms are still written as

$$
\vec{T}=\vec{R}+\vec{\varrho}_{i},
$$

where $\vec{R}$ and $\vec{\varrho}_{i}(i=1$ to $\mu$ ) denote the vectors of the Bravais lattice and the positions of the $i$ th atom within the unit cell, respectively.

Further, consider the magnetic group

$$
M=H+K\{\gamma \mid \vec{\tau}(\gamma)\} H
$$

where $H$ is a subgroup of $G$,

$$
H \subset G,
$$

$K$ denotes the operator of time inversion, and $\{\gamma \mid \vec{\tau}(\gamma)\}$ is a space group element of $G-H$.

Assume degeneracies to exist between the bands of the considered set of $\mu$ energy bands and the bands not belonging to this set. This assumption is always true since, in any metal, there are degeneracies between the bands of any selected set of energy bands and the bands outside of this set. These degeneracies are caused by symmetry and may occur at points and lines of symmetry of the Brillouin zone. Further, assume

- these degeneracies to be removed in the subgroup $H$ of $G$ (i.e., when the representations of $G$ are replaced by the subduced representations of $H$ );
- the symmetry operation $K\{\gamma \mid \vec{\tau}(\gamma)\}$ not to produce extra degeneracies between the bands of the considered set and bands outside of this set;

- unitary matrices $\boldsymbol{S}(\vec{K})$ [as defined in Eq. (4.16) of Ref. 2] to exist which satisfy the equations (4.17) and (4.28) of Ref. 2 and Eq. (7.1) of Ref. 13; and

- the positions $\vec{\rho}_{i}$ of the Wannier functions [which are determined by these matrices $\boldsymbol{S}(\vec{K})]$ to be identical with the positions of the atoms.

Then the coefficients $g_{i q}(\vec{k})$ in Eq. (1.1) may be chosen in such a way that the Wannier functions comply with the four conditions following Eq. (1.1) with the exception that they are no longer symmetry-adapted to the paramagnetic space group $G$ but only to the subgroup $H$ of $G$. That means, Eq. (1.3) is satisfied only for the elements $\alpha$ of the point group $H_{0}$ of $H$. In addition to Eq. (1.3) we have ${ }^{13}$

$$
K w_{i}\left(\gamma^{-1}\left(\vec{r}-\vec{R}-\vec{\rho}_{i}\right)\right)=\sum_{j=1}^{\mu} D_{j i}(K \gamma) w_{j}\left(\vec{r}-\vec{R}-\vec{\rho}_{i}\right)
$$

where the matrix $\left[D_{j i}(K \gamma)\right]$ is the representative of $K \gamma$ in the corepresentation of the point group

$$
M_{0}=H_{0}+K \gamma H_{0}
$$

of $M$ which is derived from the representation $D_{0}$ of $H_{0}$ in Eq. (1.3).

Since there is exactly one Wannier function at each atom, the Wannier functions may be labeled by the positions $\vec{T}$ of the atoms,

$$
w_{\vec{T}}(\vec{r}) \equiv w_{i}\left(\vec{r}-\vec{R}-\vec{\rho}_{i}\right),
$$

and the equations (1.3) and (A8) may be considerably simplified.

Applying on both sides of Eq. (1.3) the operation $\alpha$ on $\vec{r}-\vec{R}-\vec{\rho}_{i}$ we obtain

$$
w_{i}\left(\vec{r}-\vec{R}-\vec{\rho}_{i}\right)=\sum_{j=1}^{\mu} D_{j i}(\alpha) w_{j}\left(\alpha\left(\vec{r}-\vec{R}-\vec{\rho}_{i}\right)\right),
$$

and the application of the operator $P(a)$ [given in Eq. (A3)] on both sides of this equation (A11) yields the equation

$$
P(a) w_{i}\left(\vec{r}-\vec{R}-\vec{\rho}_{i}\right)=\sum_{j=1}^{\mu} D_{j i}(\alpha) w_{j}\left(\vec{r}-\vec{t}-\alpha \vec{R}-\alpha \vec{\rho}_{i}\right)
$$

which applies to all the elements $a \in H$.

As shown in Ref. 6, Eq. (A12) may also be written in the form

$$
P(a) w_{i}\left(\vec{r}-\vec{R}-\vec{\rho}_{i}\right)=\sum_{j=1}^{\mu} D_{j i}(\alpha) w_{j}\left(\vec{r}-\alpha \vec{R}-\vec{\rho}_{j}-\vec{R}_{j}(\alpha)\right)
$$


with $\vec{R}_{j}(\alpha)$ being translations of the Bravais lattice (depending on $j$ and $\alpha$ ), see Eq. (2.13) of Ref. 6 .

Comparing the Eqs. (A12) and (A13), we see that on the right hand side of Eq. (A13) there are only Wannier functions related to an atomic position $\vec{\rho}_{j}$ within the unit cell for which the translation

$$
\vec{R}_{j}(\alpha)=\alpha \vec{\rho}_{i}+\vec{t}-\vec{\rho}_{j}
$$

is a translation vector of the Bravais lattice. This cannot be true for more than one vector $\vec{\rho}_{j}$ since all the $\vec{\rho}_{j}$ are different and lie within the unit cell. It is true for exactly one $\vec{\rho}_{j}$ because $\vec{R}_{j}(\alpha)$ is a translation of the Bravais lattice if we put

$$
\vec{\rho}_{j}=\alpha \vec{\rho}_{i}+\vec{t}
$$

and $\vec{R}+\alpha \vec{\rho}_{i}+\vec{t}$ is the position of an atom since it can be generated by the application of the space group operation $\{\alpha \mid \vec{t}\}$ on the atomic position $\alpha^{-1} \vec{R}+\vec{\rho}_{i}$.

Consequently, on the right hand side of Eq. (A12) there is only one Wannier function, namely that function related to the atom at position $\alpha \overrightarrow{\rho_{i}}+\vec{t}$ within the unit cell. Hence, the sum on the right hand side of Eq. (A12) and (analogously) of Eq. (A8) consists of one summand only. The matrices $\left[D_{j i}(\alpha)\right]$ in these equations have only one non-vanishing element, say $d_{j i}(\alpha)$, in each column which satisfies the equation

$$
\left|d_{j i}(\alpha)\right|=1
$$

since the matrix $\left[D_{j i}(\alpha)\right]$ is unitary.

Hence, Eq. (A12) may be written as

$$
P(a) w_{\vec{T}}(\vec{r})=d_{\vec{T}}(\alpha) w_{\vec{T}^{\prime}}(\vec{r}) \quad \text { for } a \in H
$$

with

$$
\vec{T}^{\prime}=\alpha \vec{T}+\vec{t}
$$

and Eq. (A8) yields

$$
K P(g) w_{\vec{T}}(\vec{r})=d_{\vec{T}}(K \gamma) w_{\vec{T}^{\prime}}(\vec{r})
$$

with

$$
g=\{\gamma \mid \vec{\tau}(\gamma)\}
$$

and

$$
\vec{T}^{\prime}=\gamma \vec{T}+\vec{\tau}(\gamma)
$$

where the coefficients $d_{\vec{T}}(\alpha)$ and $d_{\vec{T}}(K \gamma)$ have the absolute value 1 ,

$$
\left|d_{\vec{T}}(\alpha)\right|=\left|d_{\vec{T}}(K \gamma)\right|=1 .
$$

It should be noted that the time inversion $K$ does not belong to $M$. Therefore, it is not possible to choose the coefficients $g_{i q}(\vec{k})$ in Eq. (1.1) in such a way that the Wannier functions satisfy an equation analogous to Eq. (A19) by application of the time inversion operator $K$ alone.
Case (b): Spin dependent Wannier functions symmetry-adapted to the paramagnetic group

\section{a. Symmetry operators}

If in Eq. (1.1) we replace the Bloch functions $\varphi_{\vec{k} q}(\vec{r})$ by Bloch functions

$$
\phi_{\vec{k} q m}(\vec{r}, t)=\sum_{s=-\frac{1}{2}}^{+\frac{1}{2}} f_{s m}(q, \vec{k}) u_{s}(t) \varphi_{\vec{k} q}(\vec{r})
$$

with $\vec{k}$ dependent spin directions, we get "spin dependent Wannier functions"

$$
\begin{aligned}
& w_{i m}\left(\vec{r}-\vec{R}-\vec{\rho}_{i}, t\right) \\
& =\frac{1}{\sqrt{N}} \sum_{\vec{k}}^{B Z} \sum_{q=1}^{\mu} e^{-i \vec{k}\left(\vec{R}+\vec{\rho}_{i}\right)} g_{i q}(\vec{k}) \phi_{\vec{k} q m}(\vec{r}, t),
\end{aligned}
$$

which are labeled by the additional quantum number $m= \pm \frac{1}{2}$ of the crystal spin. The functions $u_{s}(t)$ denote Pauli's spin functions as given in Eq. (4.7) and the coefficients $f_{s m}(q, \vec{k})$ form (for each $\vec{k}$ and $q$ ) a unitary two-dimensional matrix $\boldsymbol{f}(q, \vec{k})$,

$$
\boldsymbol{f}^{-1}(q, \vec{k})=\boldsymbol{f}^{\dagger}(q, \vec{k})
$$

If we have

$$
f_{s m}(q, \vec{k})=\delta_{s m},
$$

the two functions $\phi_{\vec{k} q m}(\vec{r}, t)$ (with $m= \pm \frac{1}{2}$ ) are usual Bloch functions with the spins lying in $+z$ and $-z$ direction, respectively. Otherwise, the functions $\phi_{\vec{k} q m}(\vec{r}, t)$ still are usual Bloch functions with antiparallel spins which, however, no longer lie in $\pm z$ direction.

As in the preceding case (a), consider a set of $\mu$ energy bands in the paramagnetic band structure of a metal with $\mu$ atoms per unit cell.

The paramagnetic group $M^{P}$ of the metal may be written as

$$
M^{P}=G+K G
$$

where $K$ still denotes the operator of time inversion. Assume

- the symmetry degeneracies between the bands belonging the considered set and bands not belonging to this set to be removed when the singlevalued representations of $G$ are replaced by the corresponding double-valued representations;

- the time inversion symmetry not to produce extra degeneracies between the bands of the considered set and bands outside of this set; 
- unitary matrices $\boldsymbol{S}(\vec{K})$ to exist which satisfy the equations (4.16), (4.17), and (4.28) of Ref. 2 and Eq. (7.1) of Ref. 13, when the single-valued representations in these equations are replaced by the corresponding double-valued representations; and

- the positions $\vec{\rho}_{i}$ of the Wannier functions to be identical with the positions of the atoms.

Then the coefficients $f_{s m}(q, \vec{k})$ and $g_{i q}(\vec{k})$ in Eqs. (A21) and (A22) may be chosen in such a way that also the spin dependent Wannier functions comply with the four conditions following Eq. (1.1). However, the coefficients $f_{s m}(q, \vec{k})$ cannot be chosen independent of $\vec{k}$ since the considered set of energy bands is isolated not before the single-valued representations of the Bloch functions are replaced by the related double-valued representations. [If the $f_{s m}(q, \vec{k})$ are independent of $\vec{k}$, the Wannier functions in Eq. (A22) are usual Wannier functions which may comply with the four conditions following Eq. (1.1) only if the considered set of energy bands is already isolated when the Bloch functions are labeled by the singlevalued representations of $G$.] As an important consequence, the operator $H^{\prime}$ does not conserve the crystal spin, see Eq. (3.12).

The symmetry of the spin dependent Wannier functions may be derived from the equations in Refs. 2, 6 and 13 in the same way as we have derived the symmetry of the magnetic Wannier functions in the preceding case (a). We now get the equations

$$
P(a) w_{\vec{T} m}(\vec{r}, t)=d_{\vec{T}}(\alpha) \sum_{m^{\prime}=-\frac{1}{2}}^{+\frac{1}{2}} d_{m^{\prime} m}(\alpha) w_{\vec{T}^{\prime} m^{\prime}}(\vec{r}, t)
$$

for $a \in G$, and

$$
K w_{\vec{T} m}(\vec{r}, t)=d_{\vec{T}}(K) \sum_{m^{\prime}=-\frac{1}{2}}^{+\frac{1}{2}} d_{m^{\prime} m}(K) w_{\vec{T} m^{\prime}}(\vec{r}, t),
$$

where

$$
w_{\vec{T} m}(\vec{r}, t) \equiv w_{i m}\left(\vec{r}-\vec{R}-\vec{\rho}_{i}, t\right)
$$

and

$$
\vec{T}^{\prime}=\alpha \vec{T}+\vec{t}
$$

The operators $P(a)$ now act on $\vec{r}$ and $t$, see Eq. (A3), the matrices $\left[d_{m^{\prime} m}(\alpha)\right]$ are the representatives of the two-dimensional double-valued representation $D_{1 / 2}$ of the three-dimensional rotation group $O(3)$, the matrix $\left[d_{m^{\prime} m}(K)\right]$ is given by

$$
\left[d_{m^{\prime} m}(K)\right]=\left(\begin{array}{rr}
0 & 1 \\
-1 & 0
\end{array}\right),
$$

[see, e.g., Table 7.15 of Ref. 21], and the c-numbers $d_{\vec{T}}(\alpha)$ and $d_{\vec{T}}(K)$ still have the absolute value 1 ,

$$
\left|d_{\vec{T}}(\alpha)\right|=\left|d_{\vec{T}}(K)\right|=1 .
$$

The equations (A25) and (A26) are already given in Ref. 10. Further, in appendix B of Ref. 10 simple equations are given to identify sets of energy bands complying with all the condition given above.

\section{b. Operators of the crystal spin}

Define the "group of the positions $\vec{\rho}_{i}$ " $G_{M}$ to consist of all the $\alpha \in G_{0}$ which satisfy the equation

$$
\alpha \vec{\rho}_{i}+\vec{\tau}(\alpha)=\vec{\rho}_{i}+\vec{R}_{i}
$$

for each $\vec{\rho}_{i}$, where $\vec{R}_{i}$ denotes a translation vector of the Bravais lattice, and define for all $\alpha \in G_{M}$ symmetry operators of the "crystal spin"

$$
M(\alpha)=P\left(\left\{E \mid \vec{R}-\vec{R}_{i}\right\}\right) P[\{\alpha \mid \tau(\alpha)\}] P(\{E \mid-\vec{R}\})
$$

which depend on the position

$$
\vec{T}=\vec{R}+\vec{\rho}_{i}
$$

of the (spin dependent) Wannier function on which they are acting.

From Eqs. (A30) and (A25) we obtain the equation

$$
M(\alpha) w_{\vec{T} m}(\vec{r}, t)=d_{\vec{T}}(\alpha) \sum_{m^{\prime}=-\frac{1}{2}}^{+\frac{1}{2}} d_{m^{\prime} m}(\alpha) w_{\vec{T} m^{\prime}}(\vec{r}, t)
$$

for $\alpha \in G_{M}$, showing that the operators $M(\alpha)$ leave unchanged the positions of the spin dependent Wannier functions.

\section{APPENDIX B: SYMMETRY OF THE FERMION OPERATORS}

\section{Adiabatic fermion operators}

The adiabatic fermion operators $c_{\vec{T} m}^{\dagger}$ and $c_{\vec{T} m}$ create and annihilate electrons in localized states represented by the the Wannier functions $w_{\vec{T}}(\vec{r}) u_{m}(t)$ [in the case (a) of magnetic Wannier functions] or $w_{\vec{T} m}(\vec{r}, t)$ [in the case (b) of spin dependent Wannier functions]. Hence, their symmetry is determined by the equations (4.6), (A17), (A19), (A25), and (A26). From these equations we get

$$
P(a) c_{\vec{T} m}^{(n) \dagger} P^{-1}(a)=d_{\vec{T}}(\alpha) \sum_{m^{\prime}=-\frac{1}{2}}^{+\frac{1}{2}} d_{m^{\prime} m}(\alpha) c_{\vec{T}^{\prime} m^{\prime}}^{(n) \dagger}
$$

with

$$
\vec{T}^{\prime}=\alpha \vec{T}+\vec{t}
$$


and

$$
K P(g) c_{\vec{T} m}^{(n) \dagger}[K P(g)]^{-1}=d_{\vec{T}}(K \gamma) \sum_{m^{\prime}=-\frac{1}{2}}^{+\frac{1}{2}} d_{m^{\prime} m}(K \gamma) c_{\vec{T}^{\prime} m^{\prime}}^{(n) \dagger}
$$

with

$$
\vec{T}^{\prime}=\gamma \vec{T}+\vec{\tau}(\gamma)
$$

where the superscript $(n)$ of the fermion operators should be disregarded in this section. In addition, the operators of the crystal spin satisfy the equation

$$
M(\alpha) c_{\vec{T} m}^{(n) \dagger} M^{-1}(\alpha)=d_{\vec{T}}(\alpha) \sum_{m^{\prime}=-\frac{1}{2}}^{+\frac{1}{2}} d_{m^{\prime} m}(\alpha) c_{\vec{T} m^{\prime}}^{(n) \dagger}
$$

for all the elements $\alpha$ of the group of the positions $G_{M}$, see Eqs. (A29) and (A30).

The coefficients $d_{\vec{T}}(\alpha)$ and $d_{\vec{T}}(K \gamma)$ still have the absolute value 1 ,

$$
\left|d_{\vec{T}}(\alpha)\right|=\left|d_{\vec{T}}(K \gamma)\right|=1,
$$

and the matrices $\left[d_{m^{\prime} m}(\alpha)\right]$ and $\left[d_{m^{\prime} m}(K \gamma)\right]$ still are representatives of the two-dimensional double-valued representation $D_{1 / 2}$ of the three-dimensional rotation group $O(3)$ and the corepresentation of $O(3)+K O(3)$ derived from $D_{1 / 2}$, respectively.

In the case (a) of the magnetic Wannier functions, Eq. (B1) is valid for all the elements $a$ in $H$,

$$
a \in H
$$

and in Eq. (B2) we have

$$
g=\{\gamma \mid \vec{\tau}(\gamma)\}
$$

In the case (b) of the spin dependent Wannier functions Eq. (B1) is valid for all the elements $a$ in $G$,

$$
a \in G
$$

and in Eq. (B2) we have

$$
g=\{E \mid \overrightarrow{0}\}
$$

where $E$ denotes the identity element of $G_{0}$. In the latter case the matrix $\left[d_{m^{\prime} m}(K)\right]$ is given in Eq. (A27).

\section{Nonadiabatic fermion operators}

Within the NHM, the Wannier functions $w_{\vec{T}}(\vec{r}) u_{m}(t)$ [in the case (a) of magnetic Wannier functions] or $w_{\vec{T} m}(\vec{r}, t)$ [in the case (b) of spin dependent Wannier functions] are replaced by nonadiabatic localized functions,

$$
\left.\begin{array}{l}
w_{\vec{T}}(\vec{r}) u_{m}(t) \\
w_{\vec{T} m}(\vec{r}, t)
\end{array}\right\} \longrightarrow\langle\vec{r}, t, \vec{q} \mid \vec{T}, m, n\rangle,
$$

having the same symmetry as the Wannier functions. However, in the case of the nonadiabatic localized states, the symmetry operators $P(a)$ act on $\vec{r}, t$, and on the new coordinate $\vec{q}$ according to

$$
\begin{aligned}
& P(a)\langle\vec{r}, t, \vec{q} \mid \vec{T}, m, n\rangle \\
& \quad=\left\langle\alpha^{-1} \vec{r}-\alpha^{-1} \vec{t}, \alpha^{-1} t, \alpha^{-1} \vec{q} \mid \vec{T}, m, n\right\rangle,
\end{aligned}
$$

where the symbol $\alpha^{-1} t$ is defined in Eq. (4.6), and the application of $K$ yields

$$
K\langle\vec{r}, t, \vec{q} \mid \vec{T}, m, n\rangle=g_{m}\langle\vec{r}, t, \vec{q} \mid \vec{T},-m, n\rangle^{*},
$$

with $g_{m}$ being given in Eq. (A6).

With these redefinitions of the symmetry operators, the symmetry of the nonadiabatic fermion operators $c_{\vec{T} m}^{n \dagger}$ is also given by the equations of the preceding appendix B 1. The superscript $(n)$ of the fermion operators in these equations shall indicate that they are valid for both the adiabatic and nonadiabatic fermion operators.
* krueger@physix.mpi-stuttgart.mpg.de

1 W. Heisenberg, Z. Phys. 49, 619 (1928).

${ }^{2}$ E. Krüger, phys. stat. sol. b 52, 215 (1972).

3 J. des Cloizeaux, Phys. Rev. 129, 554 (1963).

4 J. des Cloizeaux, Phys. Rev. 135, A685 (1964).

5 J. des Cloizeaux, Phys. Rev. 135, A698 (1964).

${ }^{6}$ E. Krüger, phys. stat. sol. b 52, 519 (1972).

7 E. Krüger, Phys. Rev. B 33, 2621 (1984).

8 E. Krüger, Phys. Rev. B 40, 11090 (1989).

9 E. Krüger, Phys. Rev. B 59, 13795 (1999).

10 E. Krüger, arXiv cond-mat, 0004324 (2000).

11 E. Krüger, phys. stat. sol. b 85, 493 (1978).

12 With free atoms, the nonadiabatic motion of the nuclei occurs in such a way that the total centre of mass stays at rest. In a solid state, consisting of bound atoms, this restriction does not hold.

13 E. Krüger, phys. stat. sol. b 61, 193 (1974).

14 N. F. Mott, Can. J. Phys. 34, 1356 (1956).

15 J. Hubbard, Proc. R. Soc. London, Ser. A 276, 238 (1963).

16 E. Krüger, phys. stat. sol. b 90, 719 (1978).

17 The statement " $E(\alpha)$ is not true for at least one $\alpha \in G_{0}$ " is the negation of " $E(\alpha)$ is true for $\alpha \in G_{0}$ ", where $E(\alpha)$ stands for any equation depending on $\alpha$. It is meaningless for how many elements of $G_{0}$ the equation $E(\alpha)$ is not true.

18 E. Krüger, arXiv cond-mat, 0004284 (2000).

19 E. Krüger, phys. stat. sol. b 156, 345 (1989).

20 J. Bardeen, L. N. Cooper, and J. R. Schrieffer, Phys. Rev. 108, 1175 (1957). 
21 C. Bradley and A.P.Cracknell, The Mathematical Theory of Symmetry in Solids (Claredon, Oxford, 1972).

${ }^{22}$ H.-W. Streitwolf, Gruppentheorie in der Festkörperphysik
(Akademische Verlagsgesellschaft Geest \& Portig KG, Leipzig, 1967). 